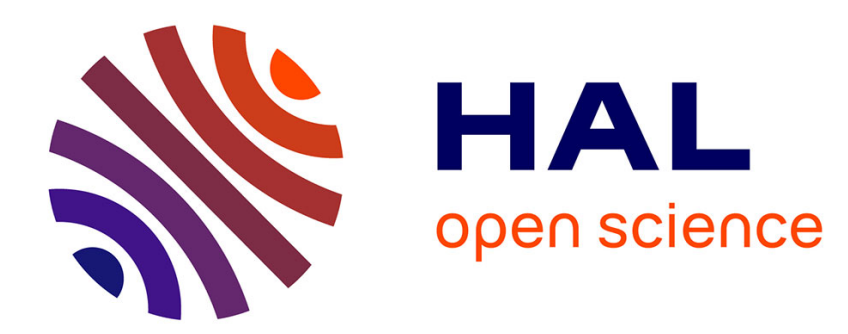

\title{
Crystallization of condensation droplets on a liquid surface
}

Olivier Pitois, Bernard François

\section{To cite this version:}

Olivier Pitois, Bernard François. Crystallization of condensation droplets on a liquid surface. Colloid and Polymer Science, 1999, 277, pp.574-578. hal-01990802

\section{HAL Id: hal-01990802 \\ https://hal.science/hal-01990802}

Submitted on 31 Jan 2019

HAL is a multi-disciplinary open access archive for the deposit and dissemination of scientific research documents, whether they are published or not. The documents may come from teaching and research institutions in France or abroad, or from public or private research centers.
L'archive ouverte pluridisciplinaire $\mathbf{H A L}$, est destinée au dépôt et à la diffusion de documents scientifiques de niveau recherche, publiés ou non, émanant des établissements d'enseignement et de recherche français ou étrangers, des laboratoires publics ou privés. 


\section{O. Pitois \\ B. François}

\section{Crystallization of condensation droplets on a liquid surface}

Received: 4 January 1999

Accepted in revised form: 15 February 1999

O. Pitois $(\square)$

Laboratoire des Matériaux et Structures

du Génie Civil

U.M.R. 113 (C.N.R.S./L.C.P.C.)

2, allée Kepler

F-77420 Champs-sur-Marne, France

e-mail: olivier.pitois@1cpc.fr

Fax: + 33-81-40 435485

B. François

Laboratoire de Recherche sur

les Matériaux Polymères

C.N.R.S./U.P.P.A.

Centre Hélioparc Pau-Pyrénées

2, avenue du Président Angot

F-64000 Pau

France
Abstract Highly ordered microporous two-dimensional membranes have been obtained from polymer solutions (Widawski et al. (1994) Nature 369: 397-399). Recently, a mechanism for the formation of such membranes was proposed, involving water vapour condensation (induced by the rapid evaporation of the volatile solvent) onto the surface of solutions and the formation of floating water droplets. Unfortunately, the droplets growth process was not observed, and consequently only qualitative information was reported. In the present paper, results of light-scattering experiments with this system are reported. The formation of water droplets growing at the surface of the solution has been observed and the evolution with time of the mean droplet radius has been found to be described by a power law with an exponent of $1 / 3$, proving that no coalescence processes occur. This particular behaviour is attributed to the precipitation of the polymer at the water/solution interface and to the formation of a mechanically resistant polymer layer encapsulating each droplet. In this way, water droplets behave like solid particles, allowing compact sheets to be formed. The presence of important surface currents is believed to promote the formation of "polycrystal" and "monocrystal" patterns.

Key words Condensation - Membrane - Scattering - Droplet Polymer

\section{Introduction}

The formation of highly ordered microporous polymeric membranes has previously been reported [1]. These membranes consist of one (or possibly several) layer(s) of spherical micron-sized pores organized in a regular triangular lattice (an example of a membrane surface is shown in Fig. 1). They are obtained by rapidly evaporating a thin layer of a solution of $\mathrm{CS}_{2}$ containing poly(p-phenylene)-block-polystyrene (PS-PPP). It was observed that the formation of the ordered structure required the presence of moisture in the surrounding atmosphere [1, 2]. Recently, a mechanism for the formation of such structures was proposed, assuming the formation of micron-sized water droplets condensed on the surface of the solution [3]. The key element in this process was found to be related to the particular surface tension properties of this system. Firstly, it was shown that the shape of a water droplet floating on the solution surface is in accordance with the pore shape observed in the membranes. In addition, PS-PPP was found to precipitate at the solution/water interface, forming a solid polymer layer around each droplet. In this way, coalescence processes are prevented, allowing the droplets to behave like solid particles trapped at a liquid interface. Then, capillary interactions and above all surface currents, contribute to organize the floating "raft" as packed sheets. In a previous paper [3], we reported optical microscopy observations of the solution surface during the condensation process. Unfortu- 


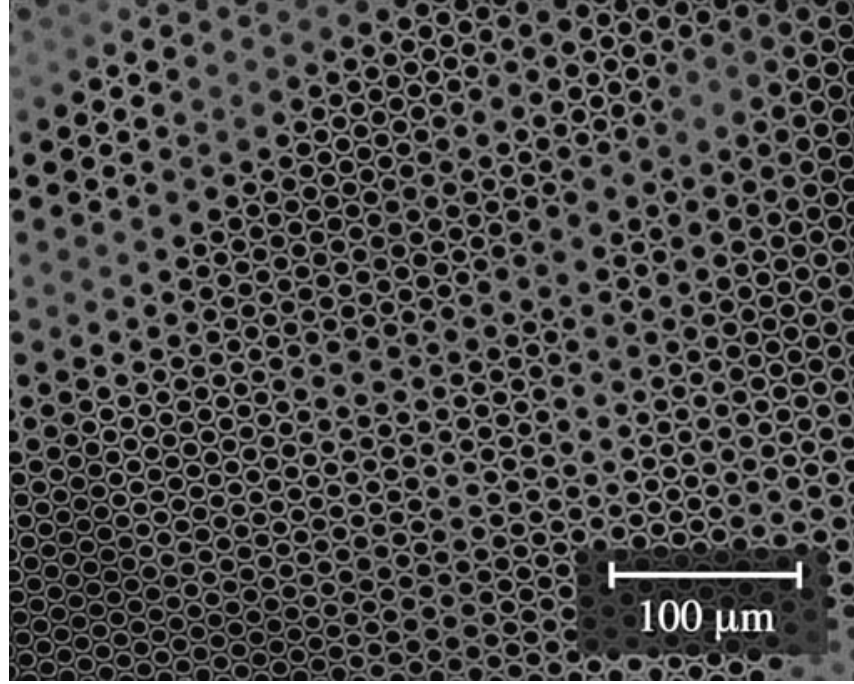

Fig. 1 Picture of a membrane surface formed from a solution of poly(p-phenylene)-block-polystyrene $\left(M_{\mathrm{w}}=3000-30000\right)$ in carbon disulphide $\left(0.02 \mathrm{~g} / \mathrm{cm}^{3}\right)$

nately, the surface was made hardly visible with high magnification because of rapid surface currents (due to solvent evaporation and to the gas flow carrying water vapour). Nevertheless, floating micron-sized droplets were distinguished under particular experimental conditions, allowing some qualitative information to be gathered about the mechanism of formation of the structure. In the present paper, we report recent results of light-scattering experiments performed with the system previously described. In contrast to previous observations in direct space, we have been able to observe the condensation process under optimum conditions for preparing well-ordered membranes. In the next section, we briefly present some theoretical aspects of correlated light scattering by a two-dimensional assembly of droplets. The experiment performed and the corresponding results are presented in subsequent sections.

\section{Scattered light by a two-dimensional assembly of droplets}

Consider $N$ droplets of radius $a$ on a surface and where the centres of the droplets are located by the position vector $\vec{r}_{i}$ with $1 \leq i \leq N$. Light of wavelength $\lambda$ and wave vector $\vec{k}_{0}$ is sent normal to the surface (see Fig. 2). The electric field and the intensity scattered can be written [4]

$\vec{E}(\theta)=\sum_{i=1}^{N} \vec{E}_{\mathrm{a}}(\theta) \cdot \exp \left[i\left(\vec{k}_{0}-\vec{k}_{\mathrm{d}}\right) \cdot \vec{r}_{i}\right]$

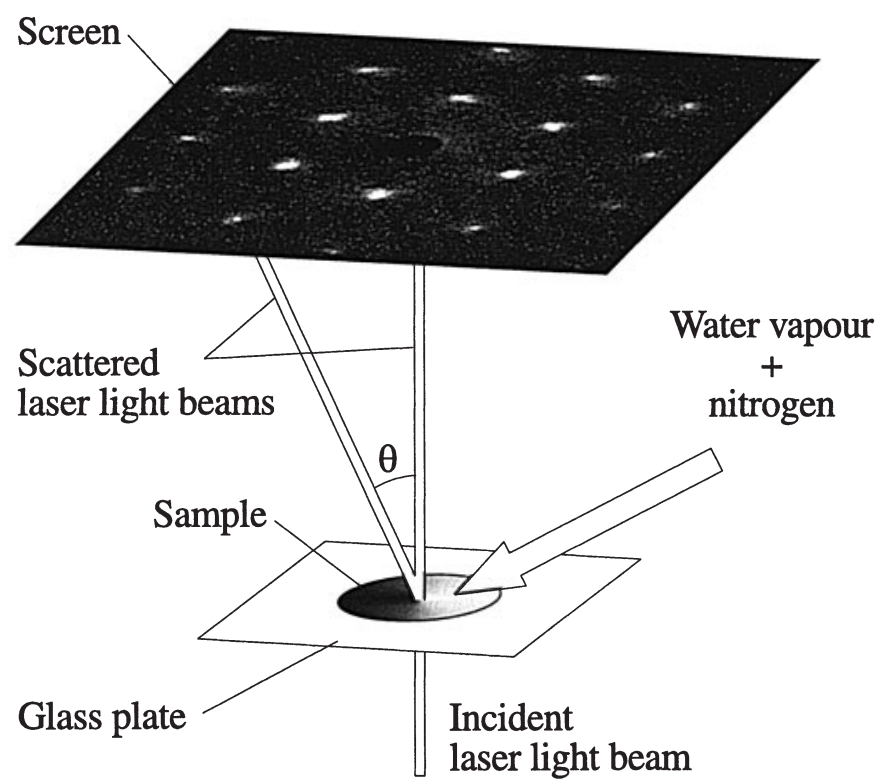

Fig. 2 Light-scattering experiment set-up. The patterns formed on the screen were filmed by means of a high-speed charge-coupled device camera

$I(\theta)=\vec{E}^{*}(\theta) \cdot \vec{E}(\theta)=I_{\mathrm{a}}(\theta) \cdot\left[N+\sum_{i=1}^{N} \sum_{\substack{j=1 \\ j \neq i}}^{N} \exp \left(i \vec{q} \cdot \vec{r}_{i j}\right)\right]$,

where $\vec{E}_{\mathrm{a}}(\theta)$ and $I_{\mathrm{a}}(\theta)$ are the electric field and the intensity scattered by one droplet, respectively, and $\vec{q}=\vec{k}_{0}-\vec{k}_{\mathrm{d}}$ is the scattering momentum transfer (where $\vec{k}_{\mathrm{d}}$ is the wave vector of the scattered light) with $|\vec{q}|=2 k \sin (\theta / 2)=(4 \pi / \lambda) \sin (\theta / 2)$. The first term in Eq. (2) represents incoherent scattering and the second accounts for the interference of radiation scattered by the droplets. Converting the sums to integrals over the surface coordinates and assuming a constant number density of droplets $(\sigma)$ over the surface, the following expression is obtained for an isotropic system [5]

$$
\begin{aligned}
I(\theta) & =N I_{\mathrm{a}}(\theta) \cdot\left\{1+2 \pi \sigma \int_{0}^{\infty} r \mathrm{~d} r[g(r)-1] \cdot J_{0}(2 k r \sin \theta)\right\} \\
& =N I_{\mathrm{a}}(\theta) S(\theta),
\end{aligned}
$$

where $J_{0}$ is the zero-order Bessel function and $S(\theta)$ is the scattering structure factor, which is, with the exception of the leading factor of unity and the number density $\sigma$, the Fourier transform of $g(r)-1$, where $g(r)$ is the pair correlation function.

\section{Experimental}

Experiments were performed under optimum conditions for preparing the membranes. A solution $(50 \mu \mathrm{l})$ was spread on a 
glass plate at room temperature. A carrier gas (nitrogen) was bubbled through a flask of distilled water at room temperature. The jet of saturated nitrogen was then sent from a nozzle onto the liquid surface (the average velocity of the gas flow was approximately $20 \mathrm{~cm} / \mathrm{s}$ ). Moisture condensation on the bottom side of the glass plate (which could induce parasite scattering during the cooling of the solution due to evaporation of the solvent) was prevented by sending dry gas onto this side. A laser beam (He-Ne, $\lambda=0.6328 \mu \mathrm{m}$, beam width $\approx 1 \mathrm{~mm}$ ) was directed towards the sample, normal to the glass plate (Fig. 2). Scattered light was projected on a screen and the patterns so formed were recorded by means of a charge-coupled device camera. The solution was composed of $\mathrm{CS}_{2}$ and PS-PPP $\left(M_{\mathrm{w}}=30000-3000,0.02 \mathrm{~g} / \mathrm{cm}^{3}\right)$. Pure solvent was also used for comparison.

\section{Results}

Four typical patterns, corresponding to four stages of the condensation process, are presented in Fig. 3. About $3 \mathrm{~s}$ after the gas flow had been sent, the first pattern to appear on the projection plane was a diffuse central peak (not presented in Fig. 3). At this stage, water droplets were randomly arranged on the surface and the surface fraction occupied by the droplets remained small. The correlation function was well approximated by $g(r)=1$ for $r>2 a$ and $I(\theta) \approx N I_{\mathrm{a}}(\theta)$. Then, the intensity of the central peak decreased strongly and spread on the disk surface (Fig. 3A). The droplets were no longer arranged randomly and so coherent scattering appeared. As $\sigma$ increased, the droplet arrangement became semi-ordered locally. At this stage $g(r \approx 2 a)>1$ and so a peak appeared in the structure function $S(\theta)$, and since the scattering system constituted by the droplets remained isotropic, a ring (first Debye-Scherrer ring) was observed on the projection plane (Fig. 3B). The maximum in the structure function rises up at

$q_{\max }=\frac{4 \pi}{\lambda} \sin \frac{\theta_{\max }}{2}=\frac{2 \pi}{2 a}$.

The patterns revealed a drastic difference between polymer solutions and pure solvent. In the first case, the sharpness of the first ring and the presence of a welldefined third ring (see Fig. 3C) prove that the assembly of droplets is a "polycrystal" [6], while only typical liquid-like scattering patterns were produced with pure solvent (the ring was generally badly defined, which accounts for the large polydispersity in the droplet radius). In the next stage, the sharp ring obtained with

Fig. 3A-D Typical scattering patterns observed during the condensation process, and corresponding to the following situations. A Small density of droplets, weak order but the droplets are no longer randomly disposed on the surface, B locally semi-ordered droplets, appearance of a characteristic length in the system, C polycrystal and D monocrystal
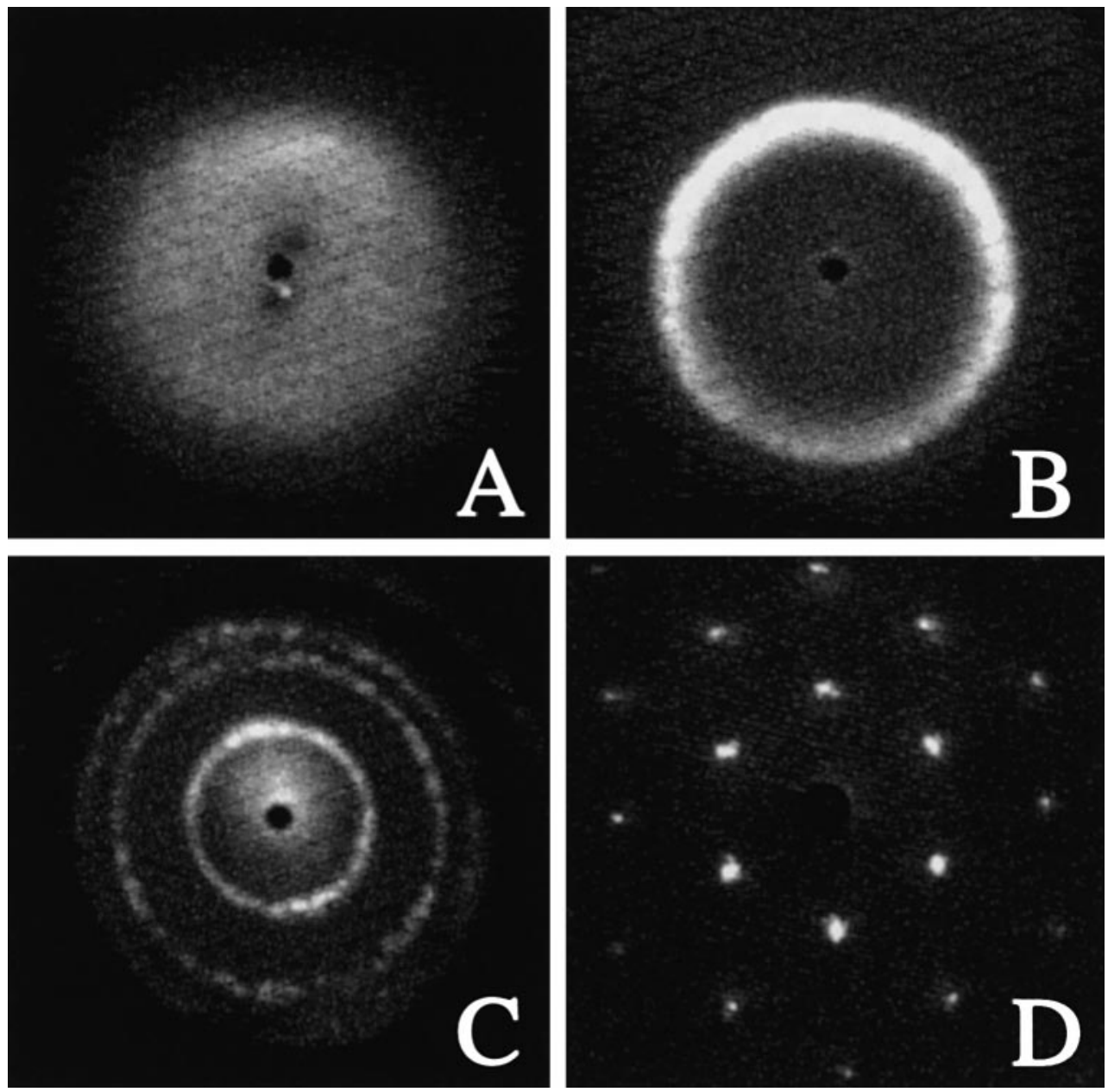
the polymer solution split into well-defined spots. Possibly, only six spots remained: monocrystallinity is thus obtained over an area equal to that of the incident laser beam (Fig. 3D). In the case of pure solvent, a sixfold modulation possibly appeared (more or less clearly) in the structure factor, but the spots remained diffuse. In contrast to polymer solutions, the structure was destroyed when the solvent completely evaporated.

In addition, it was observed that the radius of the first Debye-Scherrer ring decreased by about a factor of 2.5 during the condensation process (see Fig. 3B, C). The corresponding values of $q_{\max }$ are plotted in Fig. 4 as a function of time (the time $t=0$ refers to the appearance of a well-defined ring). It can be seen that the time evolution for $q_{\max }$ is described by a power law: $q_{\max } \sim$ $t^{-0.35}$ for times longer than $4 \mathrm{~s}$.

\section{Discussion}

From the patterns observed in Fig. 3, it appears that water vapour condenses onto the liquid surface in the shape of micron-sized droplets. The droplet growth is then observed through the evolution of the first DebyeScherrer ring. It is deduced from Fig. 4 that the time evolution for the mean droplet radius can be described by a power law with an exponent very close to $1 / 3$. This exponent was found theoretically and by experiments, in the case of single noninteracting drops condensing onto solid and liquid substrates (heterogeneous condensation) [7-11]. Note also that in the case of an assembly of interacting droplets (i.e. including coalescence processes) an exponent of unity is predicted [7]. Consequently, the condensation process in the present system can be described as the growing of noninteracting water droplets. Insofar as the exponent referring to coalescence processes $(a \sim t)$ has not been identified, even at

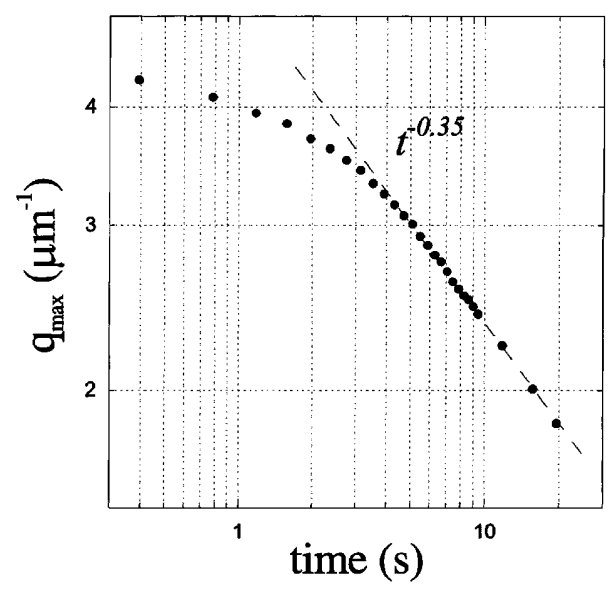

Fig. 4 Plot of $q_{\max }=2 \pi / 2 a$ (maximum in the structure function) as a function of time longer times, we are in a position to confirm our previous optical observations [3]: the solid polymer layer formed at the solution/water interface inhibits coalescence processes between droplets. It must be noted that the presence of the polymer at the interface does not affect the droplet growth. In fact, previous experiments with millimetre-sized water drops, formed in PS-PPP/ $\mathrm{CS}_{2}$ solutions by means of a syringe, revealed that the polymer coating could inhibit coalescence between two drops pressed one against the other (even when the radius of the contact area was close to the drop radius), but do not stop their growing by injection of additional amounts of water. It seems that the weak tensile strength of the polymer layer allows the increase in the surface of the drop and the creation of new portions of the solution/water interface. With regard to the variation of $a$ at shorter times, we have to note that in contrast to longer times, $\sigma$ increased in a wide range during the first stages of condensation. In addition, we expect some temperature fluctuations during this period. We refer to the following expression for the variation of $a$ as a function of the temperature difference between the gas and the substrate $\left(\Delta T_{\mathrm{s}}=T_{\text {vapour }}-T_{\text {surface }}\right)[11]$

$a \sim\left(\sqrt{U^{*}} \Delta T_{\mathrm{s}}^{0.8} D_{12} t\right)^{1 / 3}$

where $U^{*}=U \cdot\left(v D_{12}^{2}\right)^{-1 / 3}$ is the reduced flow velocity ( $U$ is the average velocity of the gas), $v$ is the kinematic viscosity and $D_{12}$ is the mutual diffusion coefficient of water in the gas. It is deduced from Eq. (5) that a power law is obtained when $\Delta T_{\mathrm{s}}$ is constant. Analysing our results with respect to Eq. (5), it seems that $\Delta T_{\mathrm{s}}$ decreases at shorter times before reaching a constant value. This behaviour was previously observed during experiments on solid surfaces [11], and was attributed to heat-transfer processes associated with the latent heat of condensation. Our case is somewhat different, in the sense that the low temperature of the liquid surface is induced by the rapid evaporation of the solvent, which is related to the "free" liquid surface of the solution. Obviously, as the "free" liquid area is reduced during the first stage of the condensation process, the rate of evaporation is strongly decreased; so, we expect the temperature of the solution surface to increase until an almost constant value for $\sigma$ is attained. The evolution of the temperature of the liquid surface seems to us hardly predictable, considering the complex heat-transfer processes inside the liquid film, but our results seem to indicate that the temperature remains at a constant value during the following stages of condensation.

Scattering patterns observed during the membrane formation are similar to those obtained with monolayers of solid particles [12-14]. The evolution of the patterns reveals the formation of compact sheets of droplets, promoted by the increase in $\sigma$ and by the presence of surface currents. Besides, capillary forces contribute to 
locally arrange the droplets in a stable hexagonal geometry so that well-ordered areas are formed. The observation of "polycrystal" patterns is in accordance with previous results obtained through optical microscopy experiments [3]; however in the present case, the experimental conditions allowed the growth of larger "crystallites" at the expense of smaller ones, and so monocrystals were obtained over large areas $\left(\approx 1 \mathrm{~mm}^{2}\right)$. It seems to us that the presence of a gas flow of relatively high velocity $(U>10 \mathrm{~cm} / \mathrm{s})$ promotes the formation of large ordered areas. The present experimental method therefore seems to be of great interest for the study of this system under optimum conditions of preparation.

\section{Conclusion}

We performed light-scattering experiments with a system composed of PS-PPP in $\mathrm{CS}_{2}$ solution. During the experiment, a flow of nitrogen saturated with water vapour was sent towards the liquid surface. The scattered light patterns have revealed that water vapour condenses in the shape of micron-sized droplets floating on the solution surface. In addition, it has been observed that the evolution with time of the mean droplet radius is described by a power law with an exponent of $1 / 3$. This value is predicted in the case of single noninteracting drops growing by heterogeneous condensation, and so our result proves that the growing droplets do not coalesce during the condensation process. This is in good accordance with the fact that the pore radius found in the resulting membrane structure is highly monodisperse. As previously proposed [3], we attribute this spectacular result to the precipitation of the polymer at the water/ solution interface and to the formation of a polymer layer encapsulating each droplet and inhibiting coalescence processes. Moreover, the establishment of the final structure has been found to result from droplet aggregation processes, promoted by important surface currents induced by the rapid evaporation of the solvent. A "polycrystal" is generally formed during the evolution of the droplet array. Finally, a hexagonal structure is obtained through the growth of "crystallites". The present experimental method appears to be an interesting tool for studying the influence of the system parameters (flow parameters, polymer concentration, etc.) on the membrane structure, and in particular on the pore size.

\section{References}

1. Widawski G, Rawiso M, François B (1994) Nature 369: 397-399

2. François B, Pitois O, François J (1995) Adv Mater 7: 1041-1044

3. Pitois O, François B Eur Physical J B (to appear in 1999)

4. Hart RW, Farell RA (1969) J Opt Soc Am 59: 766-774

5. Lock JA, Chiu C-L (1994) Appl Opt 33: 4663-4671
6. Woolhouse GR, Chaudhari P (1974) Philos Mag 31: 161-172

7. Kuz VA (1994) Phys Rev E 49: 12461250

8. Beysens D, Knobler CM (1986) Phys Rev Lett 57: 1343

9. Fritter D, Roux D, Beysens D, Knobler CM (1988) J Stat Phys 52: 1447

10. Knobler CM, Beysens D (1988) Europhys Lett 6: 707
11. Beysens D, Steyer A, Guenoun P, Fritter D, Knobler CM (1991) Phase Transitions 31: 219-246

12. Krieger IM, O’Neill F (1968) J Am Chem Soc 90: 3114

13. Goodwin JW, Ottewill RH, Parentlich A (1980) J Phys Chem 84: 15801586

14. Van Winkle DH, Murray CA (1986) Phys Rev A 34: 562-573 